\title{
BMJ Open Internet-based cognitive-behavioural therapy for tinnitus: secondary analysis to examine predictors of outcomes
}

\author{
Hansapani Rodrigo, ${ }^{1}$ Eldré W Beukes, ${ }^{2,3}$ Gerhard Andersson, ${ }^{4,5,6}$ \\ Vinaya Manchaiah (D) 2,7
}

To cite: Rodrigo H, Beukes EW, Andersson G, et al. Internetbased cognitive-behavioural therapy for tinnitus: secondary analysis to examine predictors of outcomes. BMJ Open 2021;11:e049384. doi:10.1136/ bmjopen-2021-049384

- Prepublication history and additional supplemental material for this paper are available online. To view these files, please visit the journal online. (http://dx.doi.org/10.1136/ bmjopen-2021-049384)

Received 26 January 2021 Accepted 09 August 2021

Check for updates

(C) Author(s) (or their employer(s)) 2021. Re-use permitted under CC BY-NC. No commercial re-use. See rights and permissions. Published by BMJ.

For numbered affiliations see end of article.

Correspondence to Dr Vinaya Manchaiah; vinaya.manchaiah@lamar.edu

\section{ABSTRACT}

Objectives The current study examined predictors of outcomes of internet-based cognitive-behavioural therapy (ICBT) for individuals with tinnitus.

Design Secondary analysis of intervention studies. Setting Internet-based guided tinnitus intervention provided in the UK.

Participants 228 individuals who underwent ICBT. Interventions ICBT.

Primary and secondary outcome measures The key predictor variables included demographic, tinnitus, hearing-related and treatment-related variables as well as clinical factors (eg, anxiety, depression, insomnia), which can have an impact on the treatment outcome. A 13-point reduction in Tinnitus Functional Index (TFI) scores has been defined as a successful outcome.

Results Of the 228 subjects who were included in the study, $65 \%$ had a successful ICBT outcome. As per the univariate analysis, participants with a master's degree or above had the highest odds of having a larger reduction in tinnitus severity (OR 3.47; 95\% $\mathrm{Cl} 1.32$ to 12.51), compared with the participants who had education only up to high school or less. Additionally, the baseline tinnitus severity was found to be a significant variable (OR $2.65 ; 95 \% \mathrm{Cl} 1.50$ to 4.67 ) contributing to a successful outcome with the intervention. Both linear and logistic regression models have identified education level and baseline tinnitus severity to be significant predictor variables contributing to a reduction in tinnitus severity post-ICBT. As per the linear regression model, participants who had received disability allowance had shown a 25.3-point lower TFI reduction compared with those who did not experience a decrease in their workload due to tinnitus after adjusting for baseline tinnitus severity and their education level. Conclusions Predictors of intervention outcome can be used as a means of triaging patients to the most suited form of treatment to achieve optimal outcomes and to make healthcare savings. Future studies should consider including a heterogeneous group of participants as well as other predictor variables not included in the current study. ClinicalTrial.gov Registration: NCT02370810 (completed); NCT02665975 (completed)

\section{INTRODUCTION}

Tinnitus is the perception of sounds in the absence of external stimulation and is often heard as a ringing or buzzing meaningless sound $(\mathrm{s})$. It is a very common condition with

\section{Strengths and limitations of this study}

The current study, to our knowledge, is the first study to use combined data from multiple studies to examine the predictors of internet-based cognitive-behavioural therapy (ICBT) outcome for tinnitus.

- The study included a homogeneous group of tinnitus patients due to the strict inclusion/exclusion criteria and may not have included all the possible variables (eg, health literacy, acceptability and motivation of users, satisfaction from the intervention, intervention engagement) that may have played a role in ICBT outcome.

- The sample size remained relatively small when compared with the number of predictive factors included which may have hampered the study results.

- The multivariable analyses may have some limitations in terms of examining complex relationships. Other statistical models including artificial intelligence and machine learning techniques may have more value in examining the non-linear relationship.

at least $15 \%$ of the adult population having tinnitus. ${ }^{1}$ Tinnitus is highly heterogeneous, both in the way it manifests as well as in the manner those with tinnitus respond to treatment options. ${ }^{2}$ The National Study of Hearing in England found that of the general population surveyed $(\mathrm{N}=48,313), 10.1 \%$ reported any tinnitus, $2.8 \%$ reported moderately annoying tinnitus, $1.6 \%$ reported severely annoying tinnitus and $0.5 \%$ were unable to lead a normal life due to the severity of the tinnitus. ${ }^{1}$ Although there are several management strategies described in the literature, most are not evidence based. The main exception is cognitive-behavioural therapy (CBT), as indicated in various systematic reviews of randomised controlled trials. ${ }^{3-6}$ Clinical practice guidelines based on research evidence and expert consensus recommend CBT as a management option for individuals with tinnitus and is supported by the American Academy of Otolaryngology-Head and Neck Surgery. ${ }^{7}$ 
Despite positive and strong evidence for CBT, individuals with tinnitus are rarely offered CBT in their local clinics. For example, a large-scale epidemiological study in the USA showed that medication (which is not recommended for tinnitus in clinical guidelines) was discussed $50 \%$ of the time by health professionals, whereas the CBT was discussed as a management option only $0.2 \%$ of the time. ${ }^{8}$ This is most likely a consequence of the limited number of trained professionals who provide CBT for tinnitus. One solution to overcome this issue is to use Internet-based CBT (ICBT), in which patients are provided with CBT in a self-help format over the internet with minimal guidance from a tinnitus expert. ${ }^{9}$ A series of controlled studies in Sweden, Germany the UK and the USA have demonstrated positive effects of ICBT in reducing tinnitus distress as well as reducing its comorbidities such as anxiety, depression and insomnia. ${ }^{9}$ In addition to the changes noted in standardised outcome measures, the qualitative analysis of user experiences has highlighted the perceived benefits of this programme. ${ }^{10}$ In addition, the improvements noted from ICBT have been maintained for 1-year postintervention. ${ }^{11}$ These results suggest that ICBT is a highly promising approach to provide evidence-based tinnitus management.

Although the previous studies on ICBT have shown favourable results, group effects were mainly reported. There is limited understanding of who is likely to benefit (or not) from the ICBT intervention. In other words, only a few studies have examined predictors of ICBT outcomes in tinnitus research. For example, the long-term analysis of the previous UK studies suggested that the best predictors of tinnitus improvements at 1 year were the baseline tinnitus severity, engagement with ICBT programme (ie, more modules read) and higher self-reported satisfaction with the intervention. ${ }^{11}$ Studies in other health areas have also examined the predictors of outcome for a range of internet-based health interventions. ${ }^{12-16}$ These studies have inconsistently identified various demographic as well as disease-specific variables that could predict the successful and non-successful participants on internet interventions. ${ }^{16}$ There remains a clear gap in knowledge in terms of predictors of ICBT outcomes for tinnitus.

Predictors of intervention outcomes may help triage patients to the most suitable tinnitus intervention. If interventions are recommended based on their suitability, it can potentially improve the outcomes resulting in healthcare savings. The objective of the current study was to examine the predictors of outcomes of ICBT intervention for individuals with tinnitus based on the secondary analysis of the pooled results from the three-phase clinical trial undertaken in the UK.

\section{METHOD}

\section{Study design and participants}

A large data set was sought to identify predictors of outcome. Trials with similar methodologies were hence sought to merge to form a larger data set. Although a few previous studies regarding ICBT were conducted in Europe, extensive outcome measures were not used. Following these trials, three trials were conducted in the UK using the same outcome measures. These trials were used due to a lack of other controlled trials available to pool data from. This present study, thus, formed a secondary analysis of data collected from three separate ICBT trials. Study participants from the three separate trials with different designs including the single-group pretest and post-test design, ${ }^{17}$ an efficacy RCT design ( ClinicalTrials.gov: NCT02370810), ${ }^{18}$ and an effectiveness RCT design (Clinical Trials.gov: NCT02665975) ${ }^{19}$ were combined. These studies were conducted during 2016-2018. In the efficacy trial, the experimental group underwent ICBT immediately after allocation whereas the control group underwent the same intervention following an 8-week weekly check-in period. In the effectiveness trial, the experimental group underwent the ICBT intervention whereas the control group underwent treatment as usual, consisting of in-person tinnitus counselling and sound therapy, provided by the audiologists at three hospital settings. The data were collected from only those who underwent the ICBT intervention and were included in this study. The study team was granted access to the deidentified datasets, not containing any personally identifiable information, as part of a data sharing policy. The study results are presented using the Transparent Reporting of a multivariable prediction model for Individual Prognosis or Diagnosis checklist (see online supplemental file 1 ).

Combining the data from three trials resulted in the inclusion of 228 participants. Of these, 36 were from the pilot trial, 146 were from the efficacy trial and the remaining 46 were from the effectiveness trial.

\section{Intervention}

The intervention included a CBT programme that was specifically developed for individuals with tinnitus. ${ }^{20}$ This intervention was originally developed by psychologists in Sweden, ${ }^{21}$ but later adapted by audiologists in the $\mathrm{UK}^{22}$ and the USA. ${ }^{23}$ The intervention was administered using a secured ePlatform ${ }^{24}{ }^{25}$ and presented in a self-help format. The intervention was presented over an 8 -week period, during which the users were given access to 2-3 modules each week. The CBT programme was divided into 21 modules, of which five were optional. The modules included content such as applied relaxation, thought analysis, cognitive restructuring, imagery and exposure techniques. Each module included text, images and videos to enhance the user experience. In addition, users were required to complete various exercises to engage them in the intervention. Although the intervention was presented in a self-help format, the users had access to minimal guidance from an audiologist (EWB). Generally, this included examining weekly exercises users completed and providing feedback as well as answering any questions they may have in the secured messaging system. An average of $10 \mathrm{~min}$ per participant was spent 
on providing guidance and support, although some users required more support.

\section{Outcome measures}

The study participants completed an extensive preintervention questionnaire that collected data on demographics, tinnitus-related and treatment-related history. In addition, participants also completed various standardised patient-reported outcome measures at baseline (T0), at postintervention (T1) and at the 2 month follow-up (T2). The primary outcome measure included the Tinnitus Functional Index (TFI ${ }^{26}$ to assess tinnitus severity/distress. This is a 25-item questionnaire with scores ranging between 0 and 100 . Scores below 25 indicate mild tinnitus with no need for intervention, scores ranging between 25 and 50 indicate a significant problem with possible need for intervention, and scores above 50 indicate severe enough tinnitus possibly requiring a more intensive intervention. The TFI has good psychometric properties with acceptable internal consistency (0.97) and test-retest reliability $(0.8) .{ }^{26}$

The secondary outcome measures included the Insomnia Severity Index ${ }^{27}$ as a measure of insomnia, the generalised anxiety disorder ${ }^{28}$ as a measure of anxiety, the Patient Health Questionnaire ${ }^{29}$ as a measure of depression, the Hearing Handicap Inventory for Adults Screening version $^{30}$ as a measure of self-reported hearing disability, the Hyperacusis Questionnaire ${ }^{31}$ to assess the presence hyperacusis (ie, reduced tolerance of everyday sounds), the Cognitive Failures Questionnaire ${ }^{32}$ was administered to assess cognitive functions, and the Satisfaction with Life Scales ${ }^{33}$ to assess the global life satisfaction.

\section{Patient and public involvement}

As a secondary analysis, no patients were involved in these studies. The data originate from individuals with tinnitus who had previously received CBT delivered via the internet (ie, ICBT). As the same protocol was followed for all study participants and all received the same intervention, merging this data was possible.

\section{Variables included in the predictive model Outcome variable}

The dependent variable was the pre-and post-intervention change in tinnitus distress based on the TFI score (TFI change). The 13-point change in TFI scores identified as a clinically meaningful (or significant) change by the original authors ${ }^{26}$ was used to define a clinically significant intervention outcome.

\section{Predictor variables}

Predictor variables were selected based on clinical reasoning and findings from previous studies by Beukes $e t$ $a l^{11}$ (see online supplemental file 2 for details). Thirty-two variables were selected as potential predictor (independent) variables and included demographic, tinnitus and hearing-related variables, tinnitus treatment related variables. Clinical factors are as follows:
- Demographic variables $(\mathrm{n}=7)$ : age (dichotomous), gender (dichotomous), education level (ordinal), employment type (categorical), loud noise exposure (dichotomous), diagnosed with a psychological condition (dichotomous), work less due to tinnitus (categorical).

- Tinnitus and hearing-related variables $(n=15)$ : baseline tinnitus severity (dichotomous), tinnitus duration (dichotomous), how often tinnitus heard (ordinal), tinnitus location (categorical), tinnitus types (nine different types, dichotomous), multiple tones heard (dichotomous) and hearing loss (categorical).

- Treatment-related to tinnitus $(n=4)$ : past treatment sought (dichotomous), sounds can distract from tinnitus (ordinal), hearing aid use (categorical) and medication use (dichotomous).

- Clinical factors $(\mathrm{n}=7)$ : anxiety (dichotomous), depression (dichotomous), insomnia (dichotomous), hyperacusis (dichotomous), hearing disability (dichotomous), cognitive functions (dichotomous) and life satisfaction (dichotomous).

\section{Data analysis}

The data were analysed using descriptive statistics as well as univariate and multivariable linear regression and logistic regression models. Linear models were used to identify the factors affecting a significant TFI score change while the logistic model was used to evaluate the factors which specifically effect outcomes and was thus selected. There were 98 subjects who had all their predictive variables except their post TFI scores. With the intention of preserving the power of the analysis, we have retained those subjects in the analysis after applying the predictive mean matching (PMM) data imputation. ${ }^{34}$ Data imputation with PMM has been identified to be less vulnerable to model misspecification as there is no need to define an explicit model for the distribution of the missing values. ${ }^{35}$

The univariate analysis was performed using $\chi^{2}$ or Fisher's exact test to examine the effect of single variables on the ICBT outcome using all the variables. The multivariable regression model was used to identify the effect of the variables on tinnitus reduction post ICBT while adjusting for the baseline tinnitus severity as a variable previously identified to relate to the success of ICBT. ${ }^{11}$ Prior to the multivariable analyses, the full data set was divided into training $(80 \%, \mathrm{n}=183)$ and testing $(20 \%, \mathrm{n}=45)$ to make a fair comparison among all the predictive models. The training data set was used to develop the corresponding multivariable regression models while the testing data set was used to evaluate the model predictions. Several competing multivariable models (both linear and logistic) were examined. The best models were selected based on the lowest mean squared error and the lowest Akaike information criterion (AIC). ${ }^{36}$ During multivariable analysis, we began with the full model, including all the predictor variables and used backward elimination based on AIC to select the final model. R squared and adj. $\mathrm{R}$ squared values have been reported, as they are 
Table 1 Details of clinical variables of the study participants

\begin{tabular}{ll}
\hline Characteristic & Mean (SD) \\
\hline $\begin{array}{l}\text { Preintervention tinnitus severity (measured } \\
\text { using TFI, scores range 0-11) }\end{array}$ &
\end{tabular}

Postintervention tinnitus severity (measured 34.22 (22.76) using $\mathrm{TFI}$, scores range $0-11$ )

2-month follow-up tinnitus severity
(measured using TFI, scores range 0-11)

Anxiety (measured using GAD-7, scores range 0-21)

$\begin{aligned} & \text { Depression (measured using PHQ-9, scores } \\ & \text { range 0-27) }\end{aligned}$
$\begin{aligned} & \text { Insomnia (measured using ISI, scores range } \\ & \text { 0-28) }\end{aligned}$
$\begin{aligned} & \text { Hyperacusis (measured using HQ, scores } \\ & \text { range 0-40) }\end{aligned}$
$\begin{aligned} & \text { Hearing disability (measured using the } \\ & \text { HHIA-S, scores range 0-40) }\end{aligned}$
$\begin{aligned} & \text { Cognitive failures (measured using the CFQ, } \\ & \text { scores range 0-100) }\end{aligned}$
$\begin{aligned} & \text { Life satisfaction (measured using SWLS, } \\ & \text { scores range 0-40) }\end{aligned}$

CFQ, Cognitive Failures Questionnaire; GAD-7, generalised anxiety disorder; HHIA-S, Hearing Handicap Inventory for Adults Screening ; HQ, Hyperacusis Questionnaire; ISI, Insomnia Severity Index; PHQ-9, Patient Health Questionnaire; SWLS, Satisfaction with Life Scales; TFI, Tinnitus Functional Index.

statistical measures of fit that indicate how much variation of the outcome is explained by the predictor variable(s) in a linear regression model. ${ }^{37}$ We also reported the mean squared error as it is a better measure of prediction accuracy. Both crude and model-based ORs were calculated and used to evaluate the effect of the variable. The Hosmer-Lemeshow goodness-of-fit statistic was calculated to assess the calibration of the final model. ${ }^{38}$

The dependent variable TFI change was used as a continuous variable for a linear regression analysis whereas the dichotomous variable (ie, 13-point change yes or no) was used for logistic regression analysis. All statistical analyses were performed with $\mathrm{R}$ statistical software (V.3.6.3). All tests were two sided and threshold at $5 \%$ level of significance.

\section{RESULTS}

\section{Participant demographics}

The mean age of study participants was 55.14 (SD: 12.92) years, and $57 \%$ of the subjects $(n=130)$ were males. The mean tinnitus duration was 17.68 (SD: 19.42) years. Further details on demographic, tinnitus, hearing-related and treatment-related variables are provided in online supplemental file 2 (tables 1-4). Table 1 presents details on clinical variables. The mean baseline tinnitus severity and tinnitus severity following the ICBT intervention were
TFI Score

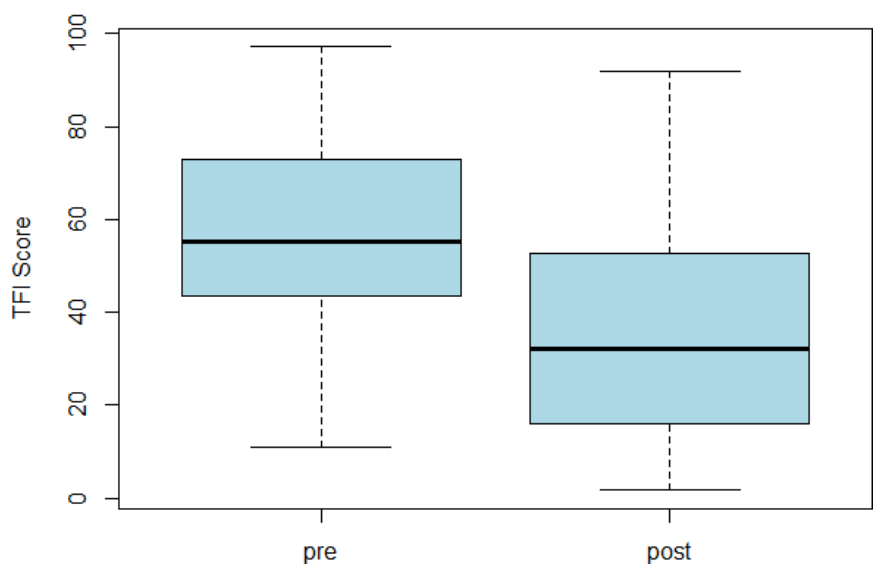

Figure 1 Tinnitus severity (TFI scores) preintervention and postintervention. Boxplot represents the five-number summary (minimum, first quartile, median, third quartile and maximum). The thick dark line represents the median. TFI, Tinnitus Functional Index.

57.93 (SD: 19.17) and 34.22 (SD: 22.76), respectively. Figure 1 presents the preintervention and postintervention tinnitus severity (TFI) score variation, indicating statistically significant differences between these scores $(\mathrm{p}<0.001)$ with the paired t-test. There were 148 participants $(65 \%)$ with a 13-point or higher reduction after the intervention.

Univariate analysis to examine the predictors of ICBT outcome With the exception of education level $(\mathrm{p}=0.01)$, none of the demographic variables were associated with postintervention tinnitus severity change of 13 points or more. Participants with a master's degree or above had the highest odds of having a larger severity change score, with an OR of 3.47 (95\% CIs 1.32 to 12.51), compared with the participants who had education only up to high school or less. In terms of tinnitus and hearing-related variables, the baseline tinnitus severity $(p=0.001)$ was significantly associated with treatment success. Participants who had a higher baseline tinnitus severity (ie, TFI scores of greater than or equal to 55.2) had significantly higher odds of treatment success (OR 2.65; 95\% CIs 1.50 to 4.67) compared with those who had a baseline severity less than 55.2. The details of the univariate analyses are provided in online supplemental file 3 (tables 1-5).

In terms of the treatment-related variables, sounds can distract $(p=0.001)$ showed a significant association with treatment success. Those who reported being distracted by the sound partially (OR 4.34; 95\% CI 1.82 to 10.34) or not at all (OR 3.15; 95\% CIs 0.99 to 10.00$)$ were at higher odds of having a successful treatment outcome when compared with those who were fully distracted. However, the odds among the participants who used hearing aids either in one ear or both ears compared with those who did not were not statistically significantly different with a $p=0.26$ (see online supplemental table 4). Tinnitus described as voice-like had a $91 \%$ lower odds of success 
Table 2 The best multiple linear regression model summary

\begin{tabular}{|c|c|c|c|}
\hline Predictor variable & Estimate & $95 \% \mathrm{Cl}$ & $P$ value \\
\hline Intercept & -28.94 & -41.70 to 16.18 & $<0.0000$ \\
\hline Work less: no & Ref & & \\
\hline Work less: stopped work & -0.58 & -10.52 to 9.36 & 0.91 \\
\hline Work less: disability allowance & -25.30 & -46.35 to 4.24 & 0.02 \\
\hline Education level: high school or less & Ref & & \\
\hline Education level: college & -2.25 & -12.61 to 8.11 & 0.67 \\
\hline Education level: vocational training & 0.98 & -10.29 to 12.25 & 0.86 \\
\hline Education level: batchelor's degree & 5.14 & -4.13 to 14.42 & 0.28 \\
\hline Education level: master's degree or above & 16.81 & 5.78 to 27.84 & 0.003 \\
\hline
\end{tabular}

Cl, Confidence Interval

with the treatment. None of the clinical factors were significantly associated with the outcome.

\section{Multivariable analyses to examine predictors of ICBT outcome}

Working less due to tinnitus $(\mathrm{p}=0.046)$, baseline tinnitus severity $(\mathrm{p}<0.001)$ and education level $(\mathrm{p}=0.014)$, showed significant associations with outcome (ie, TFI reduction). Modified models with the remaining variables were not statistically significant. Moreover, several two-way interactions were tested. We did not find any gender interactions with regard to the maskability of sounds $(p=0.87)$ and) and hearing aid usage $(p=0.68)$ variables. The overall model resulted with an $\mathrm{R}$ squared $=0.35$ and $\mathrm{Adj}$. $\mathrm{R}$ squared of 0.20 . The final model resulted in a root mean square of 22.81 on the testing data set. All required regression assumptions were satisfied with the selected model. The final regression model (see table 2) was selected with backward elimination based on AIC.

This model indicated that those who received disability allowance due to having severe tinnitus and being unable to work had shown a reduction of 25.30 points $(95 \%$ CIs -46.35 to -4.24 ) in TFI compared with those who did not have to work less due to tinnitus. Moreover, for every 10 unit increase in the baseline tinnitus severity, there was a 8.3 points (95\% CIs 0.65 to 1.00$)$ reduction in their TFI score after adjusting for other variables. Participants who had master's degree or above compared with participants who had a college education showed an expected reduction of 17 points (95\% CIs 5.78 to 27.84) in their TFI score.

Multivariable logistic regressions were performed next and indicated that baseline tinnitus severity $(\mathrm{p}<0.001)$ and education level $(\mathrm{p}=0.001)$ were identified as significant predictors (see table 3). This model had an AIC of 212.21. Modified models to the prior model indicated that other variables were not statistically significant (see table 4).

The multivariable model adjusted OR (see table 3) for the participants who had master's level or above

Table 3 The multivariable logistic regression model summary and the model adjusted OR (95\% Cl) for successful ICBT outcome of 13 points of higher

\begin{tabular}{llll}
\hline & Estimate & P value & Model-based adjusted OR (95\% CI for OR) \\
\hline Intercept & -2.32 & 0.0005 & 0.10 (0.03 to 0.37) \\
\hline Baseline tinnitus severity & 0.04 & $<\mathbf{0 . 0 0 1}$ & $\mathbf{1 . 0 4}$ (1.02 to 1.06) \\
\hline Education level: high school or less & Ref & & \\
\hline Education level: college & -0.4 & 0.41 & $0.67(0.26$ to 1.74) \\
\hline Education level: vocational training & 0.41 & 0.47 & $1.49(0.50$ to 4.48 \\
\hline Education level: batchelor's degree & 0.68 & 0.14 & $1.98(0.79$ to 4.98$)$ \\
\hline Education level: master's degree or above & 2.27 & $\mathbf{0 . 0 0 1}$ & $\mathbf{9 . 6 5}(\mathbf{2 . 3 2}$ to 40.15) \\
\hline
\end{tabular}

$\mathrm{Cl}$, Confidence Interval

; ICBT, internet-based cognitive-behavioural therapy; OR, Odds Ratio 
Table 4 Predictor variables which were insignificant in multivariable regression models

\section{$P$ value}

\section{Predictor variable}

Multivariable linear regression model

Multivariable logistic

Gender

0.47

regression model

\begin{tabular}{|c|c|c|c|}
\hline 1 & Gender & 0.47 & 0.83 \\
\hline 2 & Hearing loss & 0.89 & 0.72 \\
\hline 3 & Tinnitus type: ringing & 0.38 & 0.91 \\
\hline 5 & Tinnitus type: high pitch & 0.56 & 0.48 \\
\hline 6 & Tinnitus type: low pitch & 0.33 & 0.46 \\
\hline 8 & Tinnitus type: clicking & 0.09 & 0.01 \\
\hline 9 & Tinnitus type: music & 0.37 & 0.69 \\
\hline 10 & Tinnitus type: voices & 0.34 & 0.09 \\
\hline 11 & Tinnitus type: humming & 0.96 & 0.06 \\
\hline 12 & Anxiety & 0.07 & 0.48 \\
\hline 15 & Hyperacusis & 0.75 & 0.53 \\
\hline 16 & Hearing disability & 0.84 & 0.57 \\
\hline 17 & Cognitive functions & 0.71 & 0.72 \\
\hline 18 & Life satisfaction & 0.75 & 0.84 \\
\hline 19 & Multiple tones heard & 0.26 & 0.81 \\
\hline 20 & Loud noise exposure & 0.32 & 0.76 \\
\hline 21 & Work less due to tinnitus & Refer table 2 & 0.46 \\
\hline 22 & Presence of a psychological condition & 0.88 & 0.72 \\
\hline 23 & Past treatment sought & 0.60 & 0.83 \\
\hline 30 & Tinnitus duration & 0.17 & 0.93 \\
\hline 31 & How often tinnitus is heard & 0.23 & 0.57 \\
\hline
\end{tabular}

education compared with those who had high school education or less also showed 9.65 higher odds $(95 \%$ CIs 2.32 to 40.15 ) of having a successful outcome. Similar to the linear regression model, baseline tinnitus severity had also shown a significant association (OR 1.04; 95\% CIs 1.02 to 1.06 ) with the treatment outcome. The HosmerLemeshow goodness-of-fit test confirmed a better fit in the current model with a $p$-value of $0.50\left(\chi^{2}=7.36, \mathrm{df}=8\right)$.

Fewer variables were statistically significant in the logistic regression model, which identified influencing predictors of the ICBT success. This was due to the fact that the logistic regression model evaluated predictors of treatment successes (ie, 13-point change), while the multivariable regression model identified the predictors of a significant TFI reduction.

\section{DISCUSSION}

Accessible and affordable tinnitus interventions are needed to alleviate tinnitus distress as well as comorbid problems with anxiety, depression and insomnia. The current study examined predictors of outcomes for ICBT. In this exploratory study, only a limited number of variables were identified as possibly reducing tinnitus severity scores on the TFI by at least 13 points following ICBT intervention, and the results vary depending on the model 
used. Only educational level and baseline tinnitus severity were predictors in both linear and logistic models. The other significant variable in the linear regression models included the demographic variable, work restrictions due to tinnitus when controlling for baseline tinnitus severity and education level. These key findings are discussed below.

In terms of demographic variables, education level was found to be a significant predictor of ICBT success as those with a master's education or higher had higher odds of having a successful outcome compared with those with high school education in both the linear and logistic models. This was expected as having good literacy skills is essential when understanding the intervention materials. The intervention materials used in these studies were written at an average of ninth-grade reading level ${ }^{23}$ suggesting that they were not easily accessible for participants with only a high school education. These results highlight the importance of health literacy considerations when developing text-based self-help interventions such as ICBT. Additionally, those who reported work restrictions due to tinnitus were at a lower odds of having a successful outcome. This finding needs further exploration in future studies. Working may, for instance, provide some distraction from tinnitus as supported by reports during the 2020 COVID-19 pandemic that tinnitus was more bothersome for some individuals due to the lack of distractions from commuting and sounds at work. ${ }^{39}$ Closely monitoring the effects of tinnitus is important to ensure that tinnitus can be managed so that individuals are still able to work effectively.

When examining the tinnitus and hearing-related variables, baseline tinnitus severity was found to be a significant predictor of ICBT success, as seen in previous studies. ${ }^{11}$ Tinnitus perceptions vary greatly, and in this study, those with tinnitus presenting as musical, lower-pitched or clicking were less likely to have a positive outcome of ICBT. This finding certainly needs further exploration as the limited number of participants in each group of tinnitus perception. One of the CBT intervention aims is to help participants to reinterpret their tinnitus to a less threatening sound. It may be that these sounds are not easily likened to everyday sounds than other types of tinnitus (ie, buzzing, high pitch, pulsing, humming) making it difficult to develop adaptation strategies.

Of the four treatment-related variables, only those who reported to use of wearing one hearing aid were found to be at better odds of ICBT success. This finding needs further exploration to identify other characteristics that may be associated with an outcome such as having tinnitus in only one ear. Although the evidence for the use of hearing aids alone for tinnitus management is limited,${ }^{40}$ hearing aids may for some reduce the tinnitus percept and aid communication difficulties. ${ }^{42}$ Ensuring hearing loss is addressed in addition to the provision of ICBT may lead to more optimal outcomes for those with coexisting hearing loss.
Regarding studying the clinical factors, those with higher levels of depression were found to have a higher reduction in the TFI score. However, the participants with insomnia showed lower odds of success. Interestingly, other clinical factors including anxiety, hyperacusis, hearing disability as well as cognitive functioning were not significant predictors of ICBT in the current study. Further studies and models are required to verify these results.

Studies in other health areas have also examined the predictors of a range of internet-based health interventions. ${ }^{12-15}$ Generally, higher baseline symptoms predict increased treatment response, as in anxiety and depression, ${ }^{43}$ and higher obsessive-compulsive behaviours when treating the obsessive-compulsive disorder. ${ }^{44}$ Variables such as age and gender have been mentioned as significant predictors for some ICBT interventions. ${ }^{15} 43$ Most previous ICBT interventions have not identified pretreatment characteristics to predict or moderate outcomes. ${ }^{16}$ Most ICBT studies have indicated that ICBT works irrespective of treatment history. ${ }^{43}$ Contrarily, previous treatment has shown worse outcomes in some previous studies. ${ }^{45}$ However, it may be that some participants may have sought alternative therapies which have no evidence for tinnitus. For this reason, it would be useful to examine specific types of previous treatments in future studies to distinguish between those who had evidence-based interventions before enrolling to ICBT than those who did not.

\section{Study limitations and future research}

The current study was to our knowledge the first study to combine data from multiple studies to examine the predictors of ICBT outcome for tinnitus. However, it has limitations. First, the study may have included a homogeneous group of tinnitus patients due to study inclusion/exclusion criteria and may not have included all the possible variables (eg, health literacy, acceptability and motivation of users, satisfaction from the intervention, intervention engagement) that may have played a role in ICBT outcome. These factors were not investigated for this study. As they have been found to contribute to outcomes, ${ }^{46}$ they should be included in future studies. Second, the sample size remained relatively small when compared with the number of predictive factors included. Third, multivariable analyses may have some limitations in terms of examining complex relationships. Moreover, due to the high multicollinearity between the predictor variables, there were several competing models which had led to the same prediction accuracies and root mean square errors. Additionally, these linear models lack in identifying any predictor variables that have a non-linear relationship with the response variables. For these reasons, the study results must be viewed as preliminary. Future studies may benefit from using non-linear statistical models such as generalised additive models, and also artificial intelligence and machine learning models like neural 
networks, random forest and support vector machines, as some variables like tinnitus duration and depression had shown lower correlation with the response (with correlations: -0.10 and 0.29 , respectively). In addition, including more relevant predictive factors (eg, health literacy, motivation, engagement, adherence) in future studies may help improve predictive accuracy. Currently, we have used AIC value to compare the competing models. For future studies, we are planning to use average Area Under the Receiver Operating Characterastic curve (AUC) and Brier scores to compare models.

\section{Author affiliations}

${ }^{1}$ School of Mathematical and Statistical Sciences, The University of Texas Rio Grande Valley, Edinburg, Texas, USA

${ }^{2}$ Department of Speech and Hearing Sciences, Lamar University, Beaumont, Texas, USA

${ }^{3}$ Department of Vision and Hearing Sciences, Anglia Ruskin University, Chelmsford, UK

${ }^{4}$ Department of Behavioral Sciences and Learning, Linköping University, Linköping, Sweden

${ }^{5}$ Department of Biomedical and Clinical Sciences, Linköping University, Linköping, Sweden

${ }^{6}$ Department of Clinical Neuroscience, Division of Psychiatry, Karolinska Institute, Stockholm, Sweden

${ }^{7}$ Department of Speech and Hearing, School of Allied Health Sciences, Manipal University, Manipal, Karnataka, India

Twitter Eldré W Beukes @Eldre7, Gerhard Andersson @profGergardA and Vinaya Manchaiah @VManchaiah

Contributors HR, VM, EWB and GA conceptualised the study. EWB administered the intervention and collected the data. HR performed the data analysis. VM and HR prepared the first draft of the manuscript. All authors read and approved the final manuscript.

Funding This work was partially funded by the National Institute on Deafness and Communication Disorders (NIDCD) of the National Institute of Health (NIH) under the award number R21DC017214.

Competing interests None declared.

Patient consent for publication Not required.

Ethics approval The ethical clearance for these studies was obtained from the Faculty of Science and Technology Research Ethics Panel of Anglia Ruskin University (ARU reference: FST/FREP/14/478 and FST/FREP/14/478) and the East of England-Cambridge South Research Ethics Committee (REC reference: 16/ EE/0148) and Health Research Authority (IRAS project ID: 195565).

Provenance and peer review Not commissioned; externally peer reviewed.

Data availability statement Data are available on reasonable request.

Supplemental material This content has been supplied by the author(s). It has not been vetted by BMJ Publishing Group Limited (BMJ) and may not have been peer-reviewed. Any opinions or recommendations discussed are solely those of the author(s) and are not endorsed by BMJ. BMJ disclaims all liability and responsibility arising from any reliance placed on the content. Where the content includes any translated material, BMJ does not warrant the accuracy and reliability of the translations (including but not limited to local regulations, clinical guidelines, terminology, drug names and drug dosages), and is not responsible for any error and/or omissions arising from translation and adaptation or otherwise.

Open access This is an open access article distributed in accordance with the Creative Commons Attribution Non Commercial (CC BY-NC 4.0) license, which permits others to distribute, remix, adapt, build upon this work noncommercially, and license their derivative works on different terms, provided the original work is properly cited, appropriate credit is given, any changes made indicated, and the use is non-commercial. See: http://creativecommons.org/ licenses/by-nc/4.0/.
ORCID iD

Vinaya Manchaiah http://orcid.org/0000-0002-1254-8407

\section{REFERENCES}

1 Davis A, Refaie AE. The epidemiology of tinnitus. In: Tyler R, ed. The Handbook of tinnitus. Singular, 2020: 1-23.

2 Cederroth CR, Gallus S, Hall DA, et al. Editorial: towards an understanding of tinnitus heterogeneity. Front Aging Neurosci 2019;11:53

3 Hesser $\mathrm{H}$, Weise $\mathrm{C}$, Westin VZ, et al. A systematic review and meta-analysis of randomized controlled trials of cognitive-behavioral therapy for tinnitus distress. Clin Psychol Rev 2011;31:545-53.

4 Hoare DJ, Kowalkowski VL, Kang S, et al. Systematic review and meta-analyses of randomized controlled trials examining tinnitus management. Laryngoscope 2011;121:1555-64.

5 Landry EC, Sandoval XCR, Simeone CN, et al. Systematic review and network meta-analysis of cognitive and/or behavioral therapies (CBT) for tinnitus. Otol Neurotol 2020;41:153-66.

6 Fuller T, Cima R, Langguth B, et al. Cognitive behavioural therapy for tinnitus. Cochrane Database Syst Rev 2020;38:CD012614.

7 Tunkel DE, Bauer CA, Sun GH, et al. Clinical practice guideline: tinnitus. Otolaryngol Head Neck Surg 2014;151:S1-40.

8 Bhatt JM, Lin HW, Bhattacharyya N. Prevalence, severity, exposures, and treatment patterns of tinnitus in the United States. JAMA Otolaryngol Head Neck Surg 2016;142:959-65.

9 Beukes EW, Manchaiah V, Allen PM, et al. Internet-Based interventions for adults with hearing loss, tinnitus, and vestibular disorders: a systematic review and meta-analysis. Trends Hear 2019;23:233121651985174.

10 Beukes EW, Manchaiah V, Davies ASA, et al. Participants' experiences of an Internet-based cognitive behavioural therapy intervention for tinnitus. Int J Audiol 2018c;57:947-54.

11 Beukes EW, Allen PM, Baguley DM, et al. Long-Term efficacy of Audiologist-guided Internet-based cognitive behavior therapy for tinnitus. Am J Audiol 2018d;27:431-47.

12 Blankers M, Koeter MWJ, Schippers GM. Baseline predictors of treatment outcome in Internet-based alcohol interventions: a recursive partitioning analysis alongside a randomized trial. BMC Public Health 2013;13:455.

13 Chen H, Rodriguez MA, Qian M, et al. Predictors of treatment outcomes and adherence in Internet-based cognitive behavioral therapy for social anxiety in China. Behav Cogn Psychother 2020;48:291-303.

14 Jonas B, Tensil M-D, Leuschner F, et al. Predictors of treatment response in a web-based intervention for cannabis users. Internet Interv 2019;18:100261.

15 Spek V, Nyklícek I, Cuijpers P, et al. Predictors of outcome of group and Internet-based cognitive behavior therapy. J Affect Disord 2008;105:137-45.

16 Andersson G, Titov N, Dear BF, et al. Internet-Delivered psychological treatments: from innovation to implementation. World Psychiatry 2019:18:20-8.

17 Beukes EW, Allen PM, Manchaiah V, et al. Internet-Based intervention for tinnitus: outcome of a single-group open trial. J Am Acad Audiol 2017;28:340-51.

18 Beukes EW, Baguley DM, Allen PM, et al. Audiologist-guided Internet-based cognitive behavior therapy for adults with tinnitus in the United Kingdom: a randomized controlled trial. Ear Hear 2018;39:423-33.

19 Beukes EW, Andersson G, Allen PM, et al. Effectiveness of guided Internet-based cognitive behavioral therapy vs face-to-face clinical care for treatment of tinnitus: a randomized clinical trial. JAMA Otolaryngol Head Neck Surg 2018;144:1126-33.

20 Beukes E, Andersson G, Manchaiah V. Cognitive behavioral therapy for tinnitus. San Diego, USA: Plural Publishing Inc, 2021.

21 Andersson G, Strömgren T, Ström L, et al. Randomized controlled trial of Internet-based cognitive behavior therapy for distress associated with tinnitus. Psychosom Med 2002;64:810-6.

22 Beukes EW, Vlaescu G, Manchaiah V, et al. Development and technical functionality of an Internet-based intervention for tinnitus in the UK. Internet Interv 2016;6:6-15.

23 Beukes EW, Fagelson M, Aronson EP, et al. Readability following cultural and linguistic adaptations of an Internet-based intervention for tinnitus for use in the United States. Am J Audiol 2020;29:97-109.

24 Vlaescu G, Alasjö A, Miloff A, et al. Features and functionality of the Iterapi platform for Internet-based psychological treatment. Internet Interv 2016;6:107-14. 
25 Manchaiah V, Vlaescu G, Varadaraj S, et al. Features, functionality, and acceptability of Internet-based cognitive behavioral therapy for tinnitus in the United States. Am J Audiol 2020;29:476-90.

26 Meikle MB, Henry JA, Griest SE, et al. The tinnitus functional index: development of a new clinical measure for chronic, intrusive tinnitus. Ear Hear 2012;33:153-76.

27 Bastien $\mathrm{CH}$, Vallières A, Morin CM. Validation of the insomnia severity index as an outcome measure for insomnia research. Sleep Med 2001;2:297-307.

28 Spitzer RL, Kroenke K, Williams JBW, et al. A brief measure for assessing generalized anxiety disorder: the GAD-7. Arch Intern Med 2006;166:1092-7.

29 Spitzer RL, Kroenke K, Williams JB. Validation and utility of a selfreport version of PRIME-MD: the PHQ primary care study. primary care evaluation of mental disorders. patient health questionnaire. JAMA 1999;282:1737-44.

30 Newman CW, Weinstein BE, Jacobson GP, et al. Test-Retest reliability of the hearing handicap inventory for adults. Ear Hear 1991;12:355-7.

31 Khalfa S, Dubal S, Veuillet E, et al. Psychometric normalization of a hyperacusis questionnaire. ORL J Otorhinolaryngol Relat Spec 2002:64:436-42.

32 Broadbent DE, Cooper PF, FitzGerald P, et al. The cognitive failures questionnaire (CFQ) and its correlates. Br J Clin Psychol 1982;21:1-16.

33 Diener E, Emmons RA, Larsen RJ, et al. The satisfaction with life scale. J Pers Assess 1985;49:71-5.

34 Lodder P. To impute or not impute: That's the question. Advising on research methods: Selected topics. Huizen: Johannes van Kessel Publishing, 2013

35 Little RJA, Rubin DB. Statistical analysis with missing data. New York: John Wiley \& Sons, 2002
36 Akaike $\mathrm{H}$. A new look at the statistical model identification. IEEE Transactions on Automatic Control 1974;19:716-23.

37 Montgomery DC, Peck EA, Vinning GG. Introduction to linear regression analysis. Wiley Series in Probability and Statistics, 2012.

38 Agresti A. Categorical data analysis. 3rd edn. Hoboken: John Wiley \& Sons Inc, 2013.

39 Beukes EW, Baguley DM, Jacquemin L, et al. Changes in tinnitus experiences during the COVID-19 pandemic. Front Public Health 2020;8:592878.

40 Hoare DJ, Edmondson-Jones M, Sereda M, et al. Amplification with hearing AIDS for patients with tinnitus and co-existing hearing loss. Cochrane Database Syst Rev 2014:50:CD010151.

41 Shekhawat GS, Searchfield GD, Stinear CM. Role of hearing AIDS in tinnitus intervention: a scoping review. J Am Acad Audiol 2013;24:747-62.

42 Del Bo L, Ambrosetti U. Hearing AIDS for the treatment of tinnitus. Prog Brain Res 2007;166:341-5.

43 Stjerneklar S, Hougaard E, Thastum M. Guided Internet-based cognitive behavioral therapy for adolescent anxiety: predictors of treatment response. Internet Interv 2019;15:116-25.

44 Andersson E, Ljótsson B, Hedman E, et al. Predictors and moderators of Internet-based cognitive behavior therapy for obsessive-compulsive disorder: results from a randomized trial. $J$ Obsessive Compuls Relat Disord 2015;4:1-7.

45 Andersson G. Prior treatments in a group of tinnitus sufferers seeking treatment. Psychother Psychosom 1997;66:107-10.

46 Fuhr K, Schröder J, Berger T, et al. The association between adherence and outcome in an Internet intervention for depression. $J$ Affect Disord 2018;229:443-9. 